\title{
Pilotprojekt zur Aufarbeitung von Medikamententests
}

\author{
Marina Lienharda, Flurin Condrau ${ }^{\mathrm{b}}$ \\ ${ }^{a}$ MA, Universität Zürich, Institut für Biomedizinische Ethik und Medizingeschichte

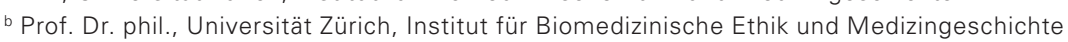

Die Medizingeschichte interessiert sich schon seit einigen Jahren für die sogenannte psychopharmakologische Wende in der Psychiatrie nach dem Zweiten Weltkrieg. Spätestens seit den 1990er Jahren hat sich die Ansicht durchgesetzt, dass die Schweizer Psychiatrie wie anderswo auch - eine erhebliche Begeisterung für die damals neuartigen Substanzen entwickelte [1].

Im Zuge der öffentlichen Aufmerksamkeit für die Geschichte von fürsorgerischen Zwangsmassnahmen im Jugendbereich rückte die Arbeit des Psychiaters Roland Kuhn, des Entdeckers des ersten Antidepressivums (Tofranil ${ }^{\circledR}$ ) in Münsterlingen, in den Vordergrund. Kuhn hatte offenbar auch vulnerable Kinder aus nahen Kinderheimen mit nicht zugelassenen Medikamenten behandelt. Medienstimmen und Betroffene erheben seit einigen Jahren schwere Vorwürfe. Eine vom Kanton Thurgau in Auftrag gegebene Studie über Kuhns Wirken steht vor dem Abschluss. Weitere Studien wurden für die Universitären Psychiatrischen Kliniken (UPK) in Basel und die Psychiatrische Universitätsklinik (PUK) in Zürich (sog. Burghölzli) erstellt [2]. Auch die Festschrift zur Geschichte der Psychiatrie Baselland (PBL) erwähnt solche Versuche, worauf die Klinikleitung einen Auftrag zu einer Pilotstudie erteilte. Nach Projektbeginn reichte Miriam Locher im

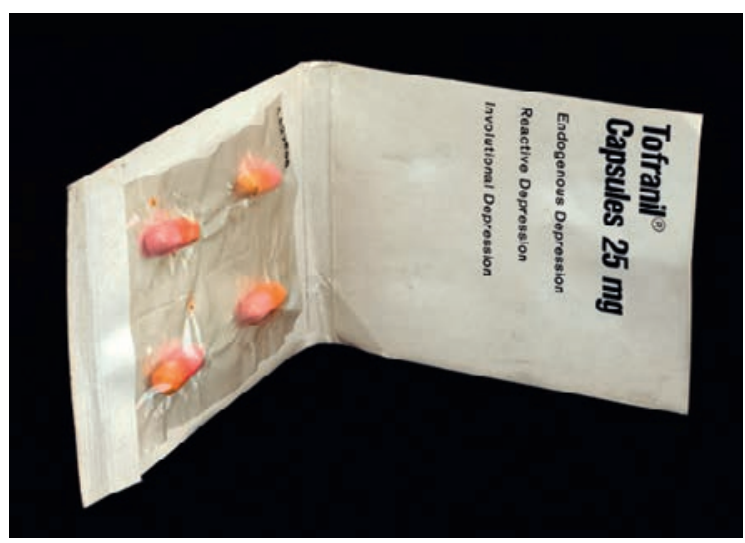

Abbildung 1: Musterpackung Tofranil-Kapseln von Geigy (UK) des Science Museum, London. Das Medikament kam 1958 auf den Schweizer Markt.
Landrat Baselland ein Postulat ein, das die Aufarbeitung der Medikamententests forderte [3]. Ziel unserer Pilotstudie war es, Art, Umfang und Rahmenbedingungen von Versuchen mit Medikamenten an der PBL ab 1950 bis 1975 erstmals genauer zu untersuchen.

\section{Die Frage der Perspektive: Historisierung von Medikamentenversuchen}

Eine grundlegende Einsicht historischen Arbeitens besteht in der konsequenten historischen Beurteilung des Untersuchungsgegenstands. Konkret: Die medizinischen, rechtlichen und ethischen Bedingungen der Medikamentenversuche zwischen 1950 und 1975 unterscheiden sich deutlich von heute. Das seit 2011 gültige Humanforschungsgesetz sowie Artikel 118b der Bundesverfassung legen fest, dass Forschung am Menschen nur durchgeführt werden darf, wenn die teilnehmenden Personen «nach hinreichender Aufklärung ihre Einwilligung erteilt haben» [4]. Für die Beurteilung der 1950er bis 1970er Jahre hilft das nicht weiter. Klare ethische oder rechtliche Kriterien zur Durchführung von Forschungsprojekten existierten damals noch nicht. Zuständig für die Zulassung neuer Medikamente waren bis zum Inkrafttreten des Heilmittelgesetzes 2002 die Kantone. Die 1934 gegründete Interkantonale Kontrollstelle für Heilmittel IKS war seit 1943 für die vorgängige Begutachtung und Registrierung zuständig [5]. Das Zulassungsverfahren war allerdings primär von der Industrie kontrolliert und zielte auf die Vertriebs- und Abrechnungsmöglichkeiten. Nach dem Contergan-Skandal begannen sich ab den frühen 1960er Jahren von den USA ausgehend verschärfte Regulierungsmechanismen für die Arzneimittelzulassung durchzusetzen. Jedoch wurden erst ab der zweiten Hälfte der 1970er Jahre in der Schweiz (und anderswo) wirksame ethische Richtlinien für Forschungen am Menschen geschaffen.

Wenn wir in diesem Text von «Medikamentenversuchen» sprechen, dann ist damit, entsprechend dem historischen Kontext, die Abgabe von Psychopharmaka zu experimentellen Zwecken - sei dies ein Heilversuch 


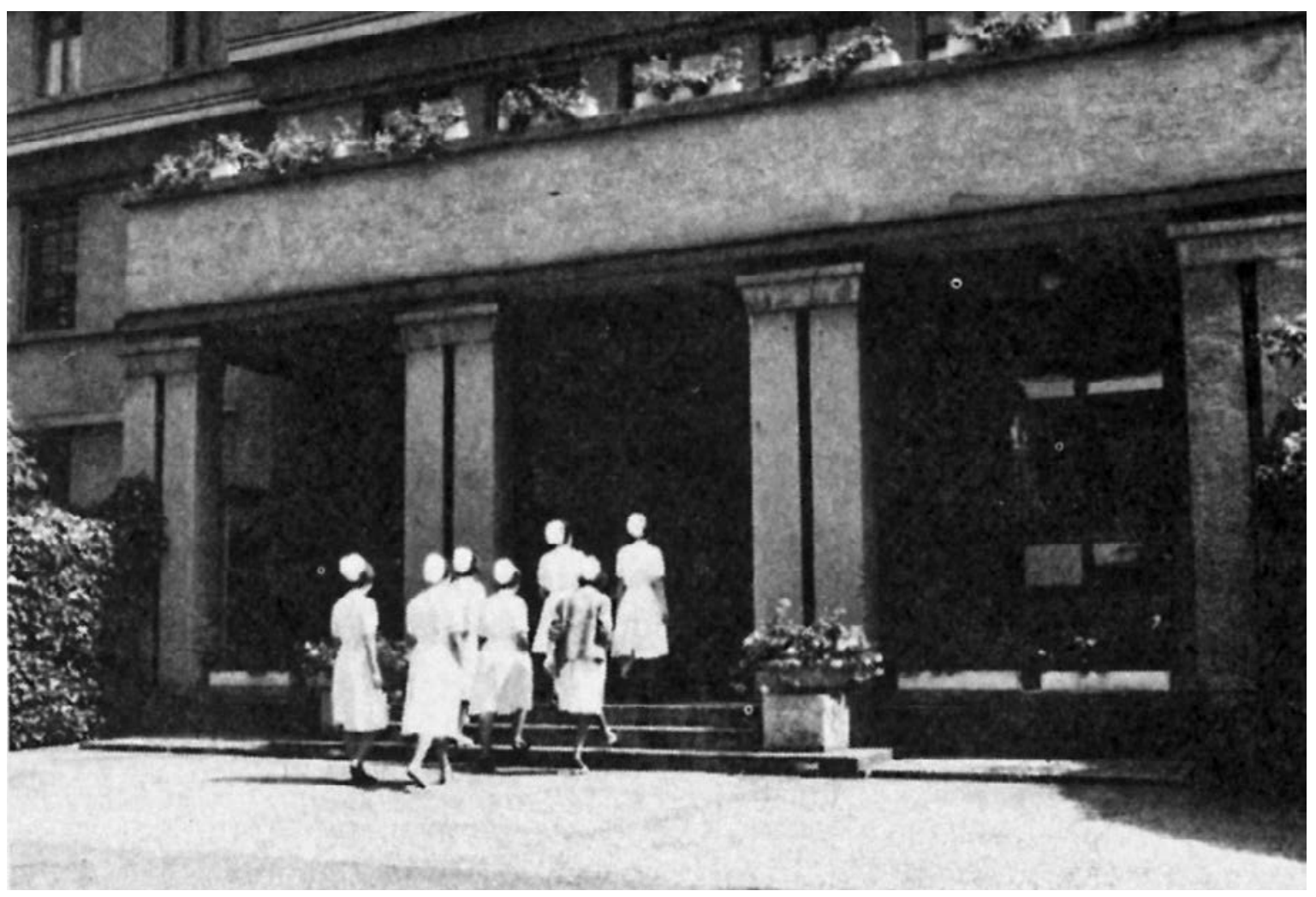

Abbildung 2: Psychiatriepflegerinnen vor dem Haus A der Psychiatrischen Klinik Hasenbühl 1965.

oder ein standardisierter Test - gemeint. Mit «Versuchspräparaten» bezeichnen wir Präparate, welche zum Zeitpunkt der Verwendung noch nicht registriert waren. Es gilt ferner zu beachten, dass die Grenzen zwischen versuchs- und standardmässig verschriebenen Präparaten zu Beginn des Untersuchungszeitraums fliessend waren.

\section{Die pharmakologische Wende an der PBL}

Im Sommer 1953 wurden in der PBL zum ersten Mal Neuroleptika eingesetzt. Es handelte sich dabei um den Wirkstoff Chlorpromazin, der unter dem Namen Largactil ${ }^{\circledast}$ von der französischen Firma Rhône-Poulenc vertrieben wurde. In den folgenden zwei Jahrzehnten wurden in rascher Abfolge weitere neue Substanzen eingeführt. Wie in anderen Psychiatrien wurde an der PBL zu Beginn der positive Einfluss der «neuen» Psychopharmaka auf die Arbeit der Pflegenden und die Atmosphäre in der Klinik gelobt. Andererseits bestand seitens der Ärzte und Ärztinnen schon von Anfang an eine gewisse Skepsis gegenüber diesen Substanzen, die im Verlauf der späten 1960er Jahre zunehmend in Ernüchterung umschlug. Sie beklagten in erster Linie die ausbleibende langfristige Heilung. Ferner zielte ihre Kritik auch auf die unerwünschten
Nebenwirkungen sowie darauf, dass es keine spezifischen Medikamente gab, welche gezielt bei einer bestimmten Diagnose eingesetzt werden konnten.

\section{Art und Umfang der Versuche}

Anhand einer zufälligen Stichprobe von 263 Krankenakten von Personen, die zwischen 1950 und 1980 stationär behandelt wurden, verschiedenen Dokumenten sowie Publikationen aus dem Verwaltungsarchiv der PBL konnten wir 16 verschiedene Präparate identifizieren, die zum Zeitpunkt ihrer Verabreichung (noch) nicht zugelassen waren und/oder im Rahmen eines Medikamentenversuchs abgegeben wurden. Viele Präparate wurden kurz vor ihrer Zulassung getestet. So zum Beispiel Chlorprothixen (Truxal ${ }^{\circledR}$ ) ab November 1959 (Zulassung 1960), Clopenthixol (Sordinol $\left.{ }^{\circledR}\right) 1961$ (Zulassung 1962) oder Opipramol (Insidon ${ }^{\circledR}$ ) 1961 (Zulassung im gleichen Jahr). Andere wurden kurz nach ihrer Zulassung geprüft, wie Chlorpromazin (Largactil ${ }^{\circledR}$ ). Das von Kuhn entdeckte Imipramin (Tofranil ${ }^{\circledR}$ ) wurde über einen langen Zeitraum getestet. Die PBL gehörte zu den ersten Kliniken, die 1955 G22150 (Iminodibenzyl-Derivat) und ab 1956 G22355 (Tofranil ${ }^{\circledR}$ ) testete, allerdings war sie bei weitem nicht die einzige: Zehn weitere Kliniken waren an den Versuchen beteiligt, und 1957 stie- 


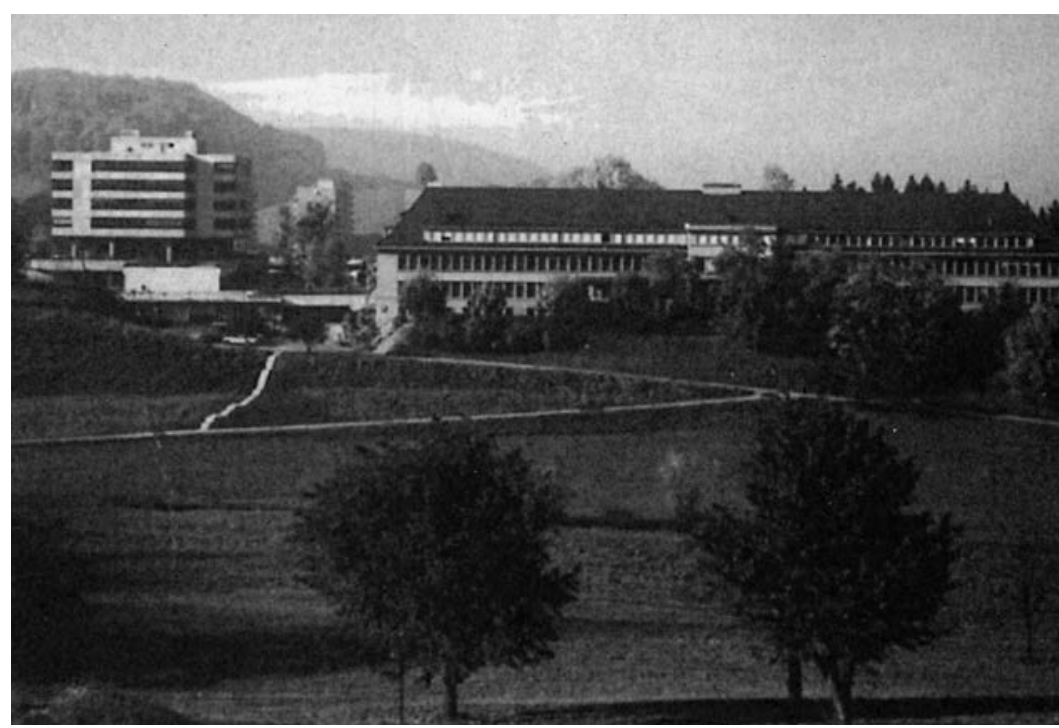

Abbildung 3: Aussenansicht der Psychiatrischen Klinik Hasenbühl (Eröffnung 1934) und des Erweiterungsbaus (Eröffnung 1974).

gen weitere zwölf in die Versuchsreihe ein. Manche getesteten Substanzen kamen unseres Wissens nie auf den Markt, wie NP207, das, wie der damalige Chefarzt Georg Stutz vor der Ärztegesellschaft 1955 erklärte, «wohl wirksam» war, «aber sehr schwere Erkrankungen der Retina hervorgerufen» hat [7]. Oder auch das ab 1964 getestete Präparat der Cilag C7245-84, die 1967 verschriebene Substanz Wy3263 oder das Geigy-Präparat G35259 (Ketimipramin). Uns erscheint es plausibel, anzunehmen, dass an der PBL eher weniger Testpräparate als an der PUK Zürich oder den UPK Basel verschrieben wurden. Nach 1972 finden sich in den von uns untersuchten Akten keine Hinweise mehr auf Testsubstanzen. Damit entsteht für uns der Eindruck, dass die Psychiatrie Baselland im Gegensatz etwa zu Münsterlingen oder Zürich eher keine Vorreiterrolle bei den Tests übernommen hat.

\section{Häufigkeit der Versuche}

Von 263 analysierten Krankenakten konnten 28 identifiziert werden, die den Einsatz von mindestens einem Präparat ohne Handelsnamen, also einem (noch) nicht zugelassenen Präparat, belegen. Das erlaubt die vorsichtige Schätzung von ungefähr $10 \%$ der Patientinnen und Patienten, denen zwischen 1953 und 1972 Testpräparate verschrieben wurden. Wir gehen ferner davon aus, dass die Versuche an der PBL ungleich über die Jahre verteilt waren. In einzelnen Stichjahren bekamen weniger als $2 \%$ der Patientinnen und Patienten Versuchspräparate. In Jahren mit grossen Versuchen wie zum Beispiel ab 1956 mit
Tofranil $^{\circledR}$ dürften mehr als $10 \%$ der stationären Patientinnen und Patienten die Substanz erhalten haben. Mit diesen Schätzungen decken sich unsere Ergebnisse mit den bereits vorliegenden Studien etwa zur PUK Zürich (2018).

Frauen sind als Versuchspatientinnen gegenüber Männern deutlich übervertreten. Dies deckt sich ebenfalls mit den Studien in Basel und Zürich. In Baselland scheinen ausserdem sogenannt chronische Patientinnen und Patienten bevorzugt mit neuen Präparaten behandelt worden zu sein. Wir fanden keine weiteren Hinweise auf eine bevorzugte Behandlung besonders vulnerabler Patientinnen- und Patientengruppen.

\section{Informed Consent oder Zwangs- massnahmen?}

Wir fanden weder in den Krankenakten noch in den Verwaltungsakten Bewilligungsformulare oder andere Hinweise darauf, dass vor der Verabreichung eines (noch) nicht zugelassenen Präparates die Zustimmung der Patientin oder des Patienten respektive der Angehörigen eingeholt wurde. Dies trifft ebenfalls für Therapien mit Standardmedikamenten zu. Wir gehen aufgrund der vergleichbaren Aktenlage in Basel-Stadt und Zürich davon aus, dass das Einholen einer formellen Einwilligung nicht zur damaligen medizinischen Praxis in der medikamentösen Therapie gehörte. Für «Elektrokuren» oder psychochirurgische Eingriffe sind hingegen Einverständniserklärungen vorhanden, da solche «Kuren» im Gegensatz zur medikamentösen Behandlung als irreversible Eingriffe galten.

Traten gravierende Nebenwirkungen auf oder zeigten die eingesetzten Arzneimittel keine Wirkung, wurden die Versuche in der Regel abgebrochen, was wir als Zeichen der Berücksichtigung des Wohls des Patienten oder der Patientin lesen. Das damalige paternalistische Arzt-Patienten-Verhältnis hatte indessen zur Folge, dass die Patientinnen und Patienten kaum Mitspracherecht hatten, was ihre Therapie betraf.

\section{Beziehungen zur Industrie}

Es sind uns keine Korrespondenzen oder andere Unterlagen zu einer möglichen Zusammenarbeit der PBL mit der Basler Chemieindustrie bekannt. Die Klinik erhielt mindestens in einem Fall von Geigy Gratismedikamente (Tofranil ${ }^{\circledR}$ ). Es ist davon auszugehen, dass dies auch bei anderen Versuchen der Fall war. Dass die PBL gelegentlich Angebote für eine Kooperation oder kleine Geschenke von Pharmafirmen erhielt, ist ebenfalls unbestritten. Es konnten aber keine Hinweise dafür gefunden werden, dass die Industrie für die Finan- 
zierung von Stellen oder Grossprojekten aufgekommen wäre. Aber selbst im Fall der PUK Zürich, wo solche Zahlungen festgestellt werden konnten, hielt die historische Studie fest, dass die Zuschüsse der Pharmaindustrie gemessen am Gesamtetat der PUK eher bescheiden waren und darüber hinaus immer geringer wurden. Für die PBL schätzen wir den Einfluss der Industrie damit insgesamt als relativ gering ein.

\section{Fazit}

Behandlungsversuche mit nicht zugelassenen Medikamenten sind an der PBL bis in die frühen 1970er Jahre nachweisbar. Den Anteil an entsprechend behandelten Patientinnen und Patienten schätzen wir auf 5-10\%. Wir gehen davon aus, dass das Einholen einer formellen Einwilligung der Patientinnen und Patienten bzw. ihrer Angehörigen für die Teilnahme an solchen Tests nicht zur damaligen medizinischen Praxis gehörte. Die PBL war eine von vielen Schweizer Kliniken, die in diesem Zeitraum Medikamente testete und nahm dabei keine Vorreiterrolle ein.

\section{Dank}

Wir möchten uns ganz herzlich bei den Mitarbeiterinnen und Mitarbeitern des Staatsarchivs Baselland für ihre tatkräftige Unterstützung bedanken.

\section{Disclosure Statement}

Dieser Bericht wurde von der Psychiatrie Baselland in Auftrag gegeben. Die PBL nahm jedoch keinen Einfluss auf die Forschung.

\section{Bildnachweise}

Abbildung 1: Science Museum, London/Wellcome Collection Abbildungen 2 und 3: Psychiatrie Baselland

\section{Literatur}

1 Healy D. The Antidepressant Era. Cambridge, MA: Harvard University Press; 1997; Brandenberger K. Psychiatrie und Psychopharmaka: Therapien und klinische Forschung mit Psychopharmaka in zwei psychiatrischen Kliniken der Schweiz, 1950-1980. Zürich; 2012 http://opac.nebis.ch/ediss/20121457.pdf; Tornay M. Zugriffe auf das Ich: Psychoaktive Stoffe und Personenkonzepte in der Schweiz, 1945 bis 1980. Mohr Siebeck; 2016; u.v.a.

2 Germann U. Medikamentenprüfungen an der Psychiatrischen Universitätsklinik Basel 1953-1980. Pilotstudie mit Vorschlägen für das weitere Vorgehen. Bern; 2017 http://www.img.unibe.ch/ unibe/portal/fak medizin/ber vkhum/inst medhist/content/ e40437/e547138/e554300/Bericht_Medikamentenprufungen_ PUK_Basel_1953-1980_ger.pdf; Rietmann T, Germann U, Condrau F. «Wenn Ihr Medikament eine Nummer statt eines Markennamens trägt». Medikamentenversuche in der Zürcher Psychiatrie 1950-1980. In: Gnädinger B, Rothenbühler V, editors. Menschen korrigieren: fürsorgerische Zwangsmassnahmen und Fremdplatzierungen im Kanton Zürich bis 1981. Zürich: Chronos 2018. p. 201-54.

3 Ott L. Man geht hinein, um wieder herauszukommen! Geschichte der Psychiatrie des Kantons Basel-Landschaft. Basel: Schwabe Verlag; 2017.

4 https://www.bv-art.ch/art-118b-forschung-am-menschen.html

5 Fischer P. Werdegang, Aufgaben und Organisation der Interkantonalen Kontrolle für Heilmittel. Interkantonale Kontrollstelle für Heilmittel (IKS), editor. 75 Jahre interkantonale Heilmittelkontrolle. Bern: Chemie-Verlag Vogt Schild AG; 1975. p. 33-86.

6 Z.B. Henckes N. Magic Bullet in the Head? Psychiatric Revolutions and their Aftermath. In: Greene JA, Condrau F, Siegel E, editors. Therapeutic revolutions: pharmaceuticals and social change in the twentieth century. Chicago; 2016. p. 65-96.

7 Stutz G. Über den Winterschlaf. Referat für die Ärztegesellschaft vom 15.12.1955, StaBL VR 3263, A.05.01. 